TRANSACTIONS OF THE

AMERICAN MATHEMATICAL SOCIETY

Volume 184, October 1973

\title{
ON THE POINTS OF WEIERSTRASS IN DIMENSIONS GREATER THAN ONE(1)
}

\author{
BY
}

ROY H. OGAWA

\begin{abstract}
In this paper, the classical concept of Weierstrass points on a Riemann surface is generalized to the consideration of similar points associated with a holomorphic vector bundle $E$ over a compact complex manifold $M$. These points are invariants of the pair $(E, M)$. The study of these general Weierstrass points is then initiated in this paper by deriving some results about the relationship of the points to singular sets of holomorphic mappings of the manifold to Grassmann spaces associated with the vector space of sections of the vector bundle. The accessibility of the point sets are demonstrated with some examples.
\end{abstract}

0. Introduction. The purpose of this paper is to generalize somewhat the definition of Weierstrass points used in the theory of compact Riemann surfaces, and the work of J. Lewittes (see [Lw]) in showing them to be related to a sequence of Kähler metrics which he constructed on Riemann surfaces.

The classical theory of Weierstrass points has played an interesting role in the study of Riemann surfaces. Especially when related to the principal mapping of Noether.

If $M$ is a compact Riemann surface of genus $g>1$, its canonical bundle $K$ has a $g$-dimensional space of sections $\Gamma(K)$. For any $p$ in $M$, we can choose a basis $\omega_{1}, \cdots, \omega_{g}$ which has orders of vanishing at $p$

$$
0=\nu_{p}\left(\omega_{1}\right)<\nu_{p}\left(\omega_{2}\right)<\cdots<\nu_{p}\left(\omega_{g}\right)<2 g-1 .
$$

$p$ is a Weierstrass point if $\nu_{p}\left(\omega_{g}\right)>g-1$. (See Gunning [G] for further details.)

These points are finite in number, the number being between $2(g+1)$ and $(g-1) g(g+1)$.

They are invariant under holomorphic automorphisms of $M$.

For each $p \in M$, there is always a section in $\Gamma(K)$ which does not vanish at $p$, and the subspace of $\Gamma(K)$ consisting of sections which vanish at $p$ has dimension $g-1$.

Consider the map of $M$ into the Grassmannian of $g-1$ dimensional

Received by the editors February 20, 1972 and, in revised form, January 22, 1973.

AMS (MOS) subject classifications (1970). Primary 32C10.

(1) This paper comprises a portion of the author's $\mathrm{Ph}$. D. Thesis, University of Calif ornia, Berkeley, 1971 . 
subspaces of $\Gamma(K)$, which is the projective space $P(\Gamma(K))$, defined by taking each $p$ in $M$ to the subspace of $\Gamma(K)$ which vanishes at $p$. This map is holomorphic and is called the principal mapping of $M$. The singularities of this mapping are the points where, in the sequence $(1), \nu_{p}\left(\omega_{2}\right)>1$. These points are certainly Weierstrass points.

The more recent work of Lewittes gave geometric meaning to the other Weierstrass points. He constructed a sequence of generalizations of the BergmanKobayashi metrics on Riemann surfaces. The $j$ th (where $1 \leq j \leq g-1$ ) is defined at points $p$ where, in the sequence (1), $\nu_{p}\left(\omega_{j}\right)=j-1$.

The points where the curvature of the metric is negative are those $p$ where in addition, in the sequence (1), $\nu_{p}\left(\omega_{j+1}\right)=j$. All other points have zero curvature.

In this paper, we generalize the definition of Weierstrass points to the situation of a compact complex manifold of dimension $n$, and an arbitrary vector bundle $E$, as long as $\Gamma(E)$ is nontrivial. This is done in $\$ 2$, after a review of the definitions and properties of jet bundles in $\$ 1$.

In $\$ 3$, we consider associated principal mappings into Grassmannians where we prove our main theorem relating the singular sets of the mappings to the sets of Weierstrass points. We point out the relationship with the work of Lewittes, a little of which was mentioned above. Actually the metrics we discuss are his curvatures. His situation on a surface lends itself to more structure for the money than we can buy in higher dimension.

In $\$ 4$, we give some examples, showing the accessibility of the points to computation.

The author wishes to acknowledge his debt to his friend and advisor, Professor S. Kobayashi.

1. The $k$-jet bundle $J^{k}(E)$. In this section we briefly review the construction that is basic to the discussions of this paper. For more details and proofs, see Chapter IV of Palais [Pa].

1.1. We use the multi-index notation throughout:

$\mu=\left(\mu_{1}, \mu_{2}, \cdots, \mu_{n}\right)$ where each $\mu_{i}$ is a nonnegative integer $z^{\mu}=z_{1}^{\mu_{1}} z_{2}^{\mu_{2}} \cdots z_{n}^{\mu_{n}}$

$d z^{\mu}=\left(d z_{1}\right)^{\mu_{1}}\left(d z_{2}\right)^{\mu_{2}} \ldots\left(d z_{n}\right)^{\mu_{n}}$ (symmetric product)

$|\mu|=\sum_{i=1}^{n} \mu_{i}$

$\mu !=\Pi_{i=1}^{n} \mu_{i}$ !

$C(\mu, \sigma)=(\mu-\sigma) ! / \mu ! \sigma !$

1.2. Let $M$ be a complex manifold of dimension $n$ and $\Omega$ be the sheaf of germs of holomorphic functions on $M$ with stalk $\Omega_{p}$ over $p$ in $M$. Let $\pi: E \rightarrow M$ 
be a holomorphic vector bundle over $M$ with fiber rank $r$ and $\Omega(E)$ be the sheaf of germs of holomorphic sections of $E$ over $M$ with stalk $\Omega_{p}(E)$ over $p$ in $M$. $\Omega(E)$ is a locally free analytic sheaf of $\Omega$-modules over $M$.

Fix an arbitrary point $p$ in $M$. Let $m_{p}$ be the unique maximal ideal of $\Omega_{p}$ consisting of all the germs which vanish at $p$, i.e. the nonunits of the integral domain $\Omega_{p}$. If $U$ is a coordinate neighborhood of $p$ with coordinate function $z=$ $\left(z_{1}, z_{2}, \ldots, z_{n}\right)$ centered at $p$, i.e. $z(p)=(0,0, \ldots, 0)$, then the elements of $\Omega_{p}$ are representable by power series $\Sigma_{|\mu| \geq 0} a_{\mu} z^{\mu}$ which converge in some neighborhood of $p$ in $U$. The elements of $m_{p}$ are those germs representable by those series of the form $\Sigma_{|\mu| \geq 1} a_{\mu} z^{\mu}$.

1.3 For integers $k \geq-1$, we consider the ideal $m_{p}^{k+1}$, where $m_{p}^{0}=\Omega_{p}$. The elements of $m_{p}^{k+1}$ are those in $\Omega_{p}$ represented by $\Sigma_{|\mu| \geq k} a_{\mu} z^{\mu}$.

Define the submodule $Z_{p}^{k}$ of $\Omega_{p}(E)$ by $Z_{p}^{k}=m_{p}^{k+1} \cdot \Omega_{p}(E)$.

Now define $J_{p}^{k}(E)=\Omega_{p}(E) / Z_{p}^{k}$ for $k \geq-1$. We are identifying germs of sections of $E$ at $p$, which, in some local representation, have the same derivatives of order less than or equal to $k$. These derivatives then give a local trivialization of $J^{k}(E)=\bigcup_{p \in M} J_{p}^{k}(E)$ which makes it a holomorphic vector bundle of fiber rank $R_{k}=r \cdot C(n+k, n)$ where $C(n+k, n)=(n+k) ! / n ! k !$. Note that $J^{-1}(E)=0$ and that $J^{0}(E)=E$.

1.4 We have the natural homomorphisms

$$
j_{p}^{k}: \Omega_{p}(E) \rightarrow J_{p}^{k}(E), j_{p}^{k}: \Omega(E) \rightarrow J_{p}^{k}(E), \text { and } j^{k}: \Gamma(E) \rightarrow \Gamma\left(J^{k}(E)\right)
$$

where $\Gamma(E)$ denotes the vector space of holomorphic sections of $E$ over $M$.

Since $m_{p}^{k} \supset m_{p}^{k+1}$, we have, for each $k \geq-1$, an induced homomorphism of vector bundles

$$
\pi^{k+1, k}: J^{k+1}(E) \rightarrow J^{k}(E),
$$

which is analytic and surjective.

1.5. Letting $T^{*}$ denote the holomorphic cotangent bundle of $M$ and $S^{k}\left(T^{*}\right)$ its kth symmetric power, we have an injection (see Palais $[\mathrm{Pa}]$ )

$$
0 \rightarrow S^{k+1}\left(T^{*}\right) \otimes E \rightarrow J^{k+1}(E) \text {. }
$$

The homomorphism is defined locally as follows: Let $p$ be a point in $M, z=$ $\left(z_{1}, z_{2}, \ldots, z_{n}\right)$ be a local coordinate system centered at $p$, and $\left\{v_{1}, v_{2}, \ldots, v_{r}\right\}$ be a local basis for $E$ near $p$. Thus each $v_{i}$ is a section of $E$ in a neighborhood of $p$ in $M$ and the set $\left\{v_{1}(q), v_{2}(q), \ldots, v_{r}(q)\right\}$ is a basis for the vector space $E_{q}$ for $q$ in some neighborhood of $p$. Map the element $\left(d z^{\mu}\right)_{p} \otimes v_{i}(p)$, where $|\mu|=$ $k+1$, of $\left(s^{k+1}\left(T^{*}\right) \otimes E\right)_{p}$ to the equivalence class in $\Omega_{p}^{p}(E) / Z_{p}^{k+1}$ represented by the local section $z^{\mu} \nu_{i}$ of $E$ in some neighborhood of $p$. Extend this map by linearity to get the desired homomorphism of vector bundles. 
Clearly (from the construction), the image of $\left(s^{k+1}\left(T^{*}\right) \otimes E\right)_{p}$ is $Z_{p}^{k} / Z_{p}^{k+1}$, so that we have the short exact sequence

$$
0 \rightarrow S^{k+1}\left(T^{*}\right) \otimes E \rightarrow J^{k+1}(E) \rightarrow J^{k}(E) \rightarrow 0 .
$$

The discussion also gives the exact sequence

$$
0 \rightarrow Z_{p}^{k+1} \rightarrow Z_{p}^{k} \rightarrow\left(S^{k}\left(T^{*}\right) \otimes E\right)_{p} \rightarrow 0 .
$$

2. Weierstrass points. Let $M$ be a compact complex manifold of dimension $n, E$ a holomorphic vector bundle on $M$ with fiber rank $r$, and $\Gamma(E)$ the vector space of holomorphic sections of $E$. Since $M$ is compact, $\Gamma(E)$ has finite dimension. We assume that the dimension of $\Gamma(E)$ is nonzero. Let $H$ be a nontrivial linear subspace of $\Gamma(E)$ of dimension $b$.

2.1. For each $p$ in $M$ and each integer $k \geq-1$, define $H_{p}^{k+1}$ to be the kernel of the kth jet evaluation map at $p, j_{p}^{k}: H \rightarrow J_{p}^{k}(E)$. We remark that this is the inverse image of $Z_{p}^{k}$ in the homomorphism $H \rightarrow \Omega_{p}(E)$ which takes the section to its germ at $p$. The definition is equivalent to the exactness of

$$
0 \rightarrow H_{p}^{k+1} \rightarrow H \rightarrow J_{p}^{k}(E)
$$

which parallels $(e 1)$.

Proposition. (i) For $k \geq-1, H_{p}^{k+1} \supset H_{p}^{k+2}$.

(ii) There is an exact sequence

$$
0 \rightarrow H_{p}^{k+2} \rightarrow H_{p}^{k+1} \rightarrow\left(S^{k+1}\left(T^{*}\right) \otimes E\right)_{p} .
$$
$\supset Z_{p}^{k+1}$.

Proof. (1) is clear from the remark after the definition and the fact that $Z_{p}^{k}$

First we define the last homomorphism in (e4) to be the dashed arrow defined by the diagram:

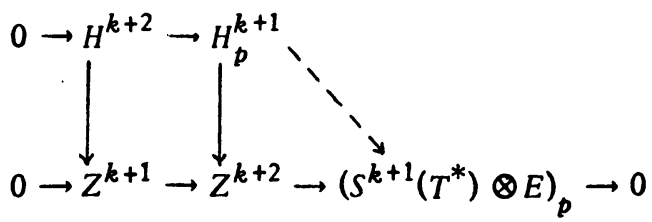

The exactness of the sequence (e4) then follows by examining the above diagram and using the remark after the definition of $H_{p}^{k+1}$. Q.E.D.

The exact sequence (e4) parallels (e2).

2.2. For those $k$, satisfying $R_{k} \leq b$ (where $R_{k}=r C(n+k, n)$ ), define

$$
W_{k}(H)=\left\{p \in M \mid j_{p}^{k}: H \rightarrow J_{p}^{k}(E) \text { is not surjective }\right\} .
$$

So for $p$ not in $W_{k}(H)$ we have the exactness of 


$$
0 \rightarrow H_{p}^{k+1} \rightarrow H \rightarrow J_{p}^{k}(E) \rightarrow 0
$$

We also have the

Proposition. For $p$ not in $W_{k}(H)$, where $k \geq 0$, we bave the exact sequence (e6)

$$
0 \rightarrow H_{p}^{k+1} \rightarrow H_{p}^{k} \rightarrow\left(S^{k}\left(T^{*}\right) \otimes E\right)_{p} \rightarrow 0 .
$$

Proof. We start with (e4) and show surjectivity of the last homomorphism. Consider the diagram

$$
\begin{gathered}
0 \\
\downarrow \\
\left(S^{k}\left(T^{*}\right) \otimes E\right)_{p} \\
\downarrow H_{p}^{k+1} \rightarrow H \stackrel{j_{p}^{k}}{\rightarrow} J_{p}^{k}(E) \rightarrow 0 \\
\downarrow \\
J_{p}^{k-1}(E) \\
\downarrow \\
0
\end{gathered}
$$

The image of $H_{p}^{k}$ by $j_{p}^{k}$ in $J_{p}^{k}(E)$ is identical with the image of $\left(S^{k}\left(T^{*}\right) \otimes E\right)_{p}$ by the injection. Q.E.D.

For those $k$, satisfying $R_{k}>b$, define

$$
W_{k}(H)=\left\{p \in M \mid j_{p}^{k}: H \rightarrow J_{p}^{k}(E) \text { is not injective }\right\} \text {. }
$$

Generally then we will say that $w_{k}(H)$ is the set of $p$ in $M$ where $j_{p}^{k}: H \rightarrow J_{p}^{k}(E)$ is not of maximal rank. $W_{k}(H)$ is called the $k$ th order Weierstrass set for $M, E$, and $H$.

2.3. Corollary. (i) $W_{-1}(H)=\varnothing$.

(ii) If $R_{k}<R_{k+1} \leq b$, then $W_{k}(H) \subset W_{k+1}(H)$.

(iii) If $b<R_{k}<R_{k+1}$, then $W_{k}(H) \supset W_{k+1}(H)$.

(iv) If $H_{1} \subset H_{2}$ and $R_{k} \leq b_{1}<b_{2}$, then $W_{k}\left(H_{1}\right) \supset W_{k}\left(H_{2}\right)$.

(v) If $H_{1} \subset H_{2}$ and $b_{1}<b_{2}<R_{k}$, then $W_{k}\left(H_{1}\right) \subset W_{k}\left(H_{2}\right)$.

Proof. Since $J_{p}^{-1}(E)=0$, (i) is obvious.

The diagram

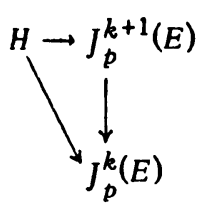

gives (ii) and (iii) immediately. 
The diagram

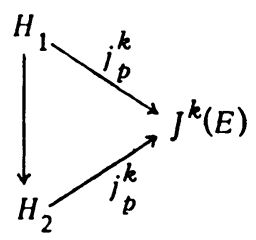

gives (iv) and (v) immediately. Q.E.D.

Thus if $k^{\prime}$ is the greatest integer satisfying $R_{k}{ }^{\prime} \leq b$, we have the two chains

$$
\varnothing=W_{-1}(H) \subset W_{0}(H) \subset \ldots \subset W_{k}^{\prime}(H)
$$

and

$$
w_{k^{\prime}+1}(H) \supset w_{k^{\prime}+2}(H) \supset \ldots
$$

It is quite clear that there need be no inclusion relationship between $W_{k} \cdot(H)$ and $W_{k^{\prime}+1^{\prime}}(H)$.

2.4. Proposition. The $W_{k}(H)$ are analytic subvarieties of $M$.

Proof. In fact, consider the trivial bundle $M \times H$ and the obvious bundle map $j^{k}: M \times H \rightarrow J^{K}(E)$. Then for $m=\min \left(R_{k}, b\right)$, take the $m$ th exterior power bundle map $\bigwedge^{m} j^{k}: \bigwedge^{m}(M \times H) \rightarrow \bigwedge^{m} J^{k}(E)$. This gives a section $\bigwedge^{m} j^{k}$ of the bundle

$$
\operatorname{Hom}\left(\bigwedge^{m}(M \times H), \bigwedge^{m} J^{k}(E)\right) \simeq\left(\bigwedge^{m}(M \times H)\right)^{*} \otimes \bigwedge^{m} J^{k}(E)
$$

whose zero set is $W_{k}(H)$. (The asterisk denotes the dual bundle.) Q.E.D.

2.5. We remark that the sets $W_{k}(H)$ have a finer structure. For each $k$ and integer $u$ satisfying $0 \leq u \leq R_{k}$, define

$$
W_{k}^{u}(H)=\left\{p \in M \mid \operatorname{rank}\left(j_{p}^{k}: H \rightarrow J_{p}^{k}(E)\right)<\min (u, b)\right\} .
$$

Then $W_{k}(H)=W_{k}^{u}(H)$ for $u=R_{k}$, and $W_{k}^{u}(H) \subset W_{k}^{u+1}(H)$ for $u<u+1 \leq R_{k}$.

Proposition. The $W_{k}^{u}(H)$ are analytic subvarieties of $M$.

Proof. As above, $w_{k}^{u}(H)$ is the zero set of the section

$$
\bigwedge^{m} j^{k} \in \Gamma\left(\left(\bigwedge^{m}(M \times H)\right)^{*} \otimes \bigwedge^{m} J^{k}(E)\right)
$$

where $m=\min (u, b)$. Q.E.D.

3. Maps into Grassmannians, semi-Kähler metrics. In this section, we again assume that we have a compact complex manifold $M$, a holomorphic vector bundle $E$ with nontrivial vector space of holomorphic sections $\Gamma(E)$, and a nontrivial subspace $H$ of $\Gamma(E)$. We then construct a mapping of $M-W_{k}(H)$ into a Grassmann 
space associated with $H$. By pulling back to $M-W_{k}(H)$, any metric in the Grassmann space then gives a semimetric on $M-W_{k}(H)$.

3.1. First, we need notation for Grassmann spaces. Let $V$ be a vector space of dimension $b$. Let $x, y$ be nonnegative integers with $x+y=b$. Let $\mathrm{Gr}_{x, y}(V)$ be the Grassmann manifold of $x$ dimensional subspaces of $V$.

We construct the standard atlas on $\mathrm{Gr}_{x, y}(V)$. Let $V^{\prime}$, and $V^{\prime \prime}$ be complementary subspaces of $V$ of dimensions $x$, and $y$ respectively, so that $V=V^{\prime} \oplus V^{\prime \prime}$. Consider the set $U_{V^{\prime}}, V^{\prime \prime}$ of $x$ dimensional subspaces $S$ of $V$ such that the projection of $V$ onto $V^{\prime}$ maps $S$ bijectively onto $V^{\prime}$. Then assign to each such $S$ the element in the space $L\left(V^{\prime}, V^{\prime \prime}\right)$ of linear mappings of $V^{\prime}$ into $V^{\prime \prime}$ which is the composition of the linear mapping $V^{\prime} \rightarrow S$, inverse to the is omorphism given by the projection of $V$ onto $V^{\prime}$, and the linear mapping $S \rightarrow V^{\prime \prime}$ which is the restriction of the projection of $V$ onto $V^{\prime \prime}$. This mapping $U_{V^{\prime}} V^{\prime \prime} \rightarrow L\left(V^{\prime}, V^{\prime \prime}\right) \cong$ $V^{\prime *} \otimes V^{\prime \prime}$, together with any isomorphism of $V^{\prime *} \otimes V^{\prime \prime}$ with $\mathrm{C}^{x y}$ gives a chart for an atlas for $\mathrm{Gr}_{x, y}(V)$, the other charts coming from varying $V^{\prime}$ and $V^{\prime \prime}$, making it a compact complex manifold of dimension $x y$.

Let $B_{x, y}(V)$ be the vector bundle of rank $x$ over $\mathrm{Gr}_{x, y}(V)$ whose points consist of pairs $(S, v)$, in the trivial bundle $G_{x, y}(V) \times V$, for which $v$ is in $S$. This is the so called tautological vector bundle over $\mathrm{Gr}_{x, y}(V)$. Letting $Q_{x, y}(V)$ denote the quotient bundle $\mathrm{Gr}_{x, y}(V) \times V / B_{x, y}(V)$, we get the following bundle exact sequence

$$
0 \rightarrow B_{x, y}(V) \rightarrow \mathrm{Gr}_{x, y}(V) \times V \rightarrow Q_{x, y}(V) \rightarrow 0
$$

over $\mathrm{Gr}_{x, y}(V)$, called the canonical sequence.

3.2. If $V$ is equipped with a hermitian metric, then $\mathrm{Gr}_{x, y}(V)$ is given a Kähler metric defined by the fundamental form $\omega$ which is defined as follows. We write the representative of $\omega$ in $U_{V^{\prime}}, V^{\prime \prime}$ by choosing orthonormal bases for $V^{\prime}$ and $V^{\prime \prime}$ and then defining

$$
\omega_{V^{\prime}, V^{\prime \prime}}(S)=-4 \sqrt{-1} \partial \bar{\partial} \log \operatorname{det}\left(I V^{\prime}+{ }^{t} \bar{Z}^{\prime}(S) Z^{\prime}(S)\right)
$$

where $I_{V^{\prime}}$ is the identity matrix, $Z^{\prime}(S)$ is the matrix of the map $Z(S): V^{\prime} \rightarrow V^{\prime \prime}$ and ${ }^{t} \bar{Z}^{\prime}(S)$ is the matrix of the adjoint of $Z(S)$, the matrices being representatives with respect to the chosen bases. This $C^{\infty}$ form $\omega$ of type (1.1) is independent of choice of orthonormal basis, is positive definite, and defines a Kähler metric on $\mathrm{Gr}_{x, y}(V)$.

3.3. Return now to the consideration of an $n$-dimensional compact complex manifold $M$, a vector bundle $E$ of rank $r$ over $M$, the vector space $\Gamma(E)$ of holomorphic sections of $E$ over $M$, which we assume to be nontrivial, and a subspace $H$ of $\Gamma(E)$ of dimension $b \neq 0$.

For each $k$ with $R_{k}<b$ and $p$ in $M-W_{k}(H)$ we have the $b-R_{k}$ dimensional 
subspace $H_{p}^{k+1}$ of $H$. This defines on the one hand, a holomorphic vector bundle $H^{k+1}$ of rank $b-R_{k}$ over $M-W_{k}(H)$, whose fiber over $p$ in $M-W_{k}(H)$ is $H_{p}^{k+1}$, and on the other hand a holomorphic mapping

$$
N_{k}(H): M_{0}-W_{k}(H) \rightarrow \mathrm{Gr}_{b-R_{k}, R_{k}}(H)
$$

called the $k$ th associated principal mapping. We have the following commutative diagram:

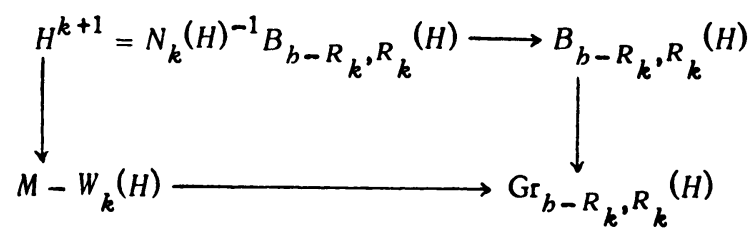

3.4. At the points in $M-W_{k}(H)$ where $N_{k}(H)$ is an immersion, any metric on the image will be pulled back to a metric.

Theorem 3.1. For $k \geq 0$, with $R_{k+1} \leq b$, let $S_{k}(H)$ be the set of singularities of the map $N_{k}(H)$. Then we have the inclusion $S_{k}(H) \subset w_{k+1}(H)$.

Proof. Let $p$ be a point of $M-W_{k+1}(H)$. We examine $d N_{k}(H)(p)$.

Choose a coordinate system in a neighborhood $G$ of $p$, centered at $p$. Thus' $z=\left(z_{1}, z_{2}, \ldots, z_{n}\right)$ is defined in $G$ and $z(p)=(0,0, \ldots, 0)$.

Since $p$ is in $M-W_{k+1}(H)$, it is in $M-W_{m}(H)$ for all $0 \leq m \leq k+1$, in particular, for $m=0$. Thus there are sections $\Phi_{1}, \cdots, \Phi_{r}$ or $E$ such that the values $\Phi_{1}(p), \ldots, \Phi_{r}(p)$ are linearly independent in $E_{p}$ and gives a basis of $E_{p}$. This is an open condition so that $\Phi_{1}(q), \ldots, \Phi_{r}(q)$ gives a basis of $E_{q}$ for all $q$ in some neighborhood of $p$. Let $G$ be made smaller so that this is true in $G$.

Choose a basis for $H$ in the following manner. First include the elements $\Phi_{1}, \ldots, \Phi_{r} \cdot$ Again, all of the $j_{p}^{m}: H \rightarrow J_{p}^{m}(E)$ are surjective for $m, 0 \leq m \leq k+1$, since $p$ is not in $w_{k+1}(H) \supset W_{k}(H) \supset \ldots \supset w_{0}(H)$. For all such $m$ then, we have (eG)

$$
0 \rightarrow H_{p}^{m+1} \rightarrow H_{p}^{m} \rightarrow\left(S^{m}\left(T^{*}\right) \otimes E\right)_{p} \rightarrow 0 \text {. }
$$

For each such $m$ then, we can choose $\Phi_{R_{m-1+1}}^{\prime}, \Phi_{R_{m-1+2}}^{\prime}, \ldots, \Phi_{R_{m}}^{\prime}$ to be elements of $H_{p}^{m}$ whose images in $\left(S^{m}\left(T^{*}\right) \otimes E\right)_{p}$ are the elements $\left(d z^{\mu}\right)_{p}^{m} \otimes \Phi_{i}(p)$ for all $\mu$ with $|\mu|=m$ and $1 \leq i \leq r$, ordered lexicographically in the $(n+2)$ tuple $(i,|\mu|, \mu)$.

Then define $\Phi_{R_{k+1}}, \Phi_{R_{k+2}}, \ldots, \Phi_{R_{k+1}}$ to be

$$
\Phi_{R_{k}+1}^{\prime}, \Phi_{R_{k}+2}^{\prime}, \ldots, \Phi_{R_{k+1}^{\prime}}^{\prime}
$$

Next, starting with $m=k$ and decreasing $m$ until $m=1$, define for $u$, $R_{m-1}+1 \leq u \leq R_{m}$ 


$$
\Phi_{u}=\Phi_{u}^{\prime}-\sum a_{u v} \Phi_{v}
$$

where the summation is over all $v, R_{m} \leq v \leq R_{k+1}$ and the $a_{u v}$ are the constants chosen so that if $u$ corresponds to the $(n+2)$-tuple $(i, m, \mu)$ then $\Phi_{u}-z^{\mu} \Phi_{i}$ is in $H_{p}^{k+2}$. (In fact if $v$ corresponds to the $(n+2)$-tuple $(j,|\nu|, \nu)$, then $a_{u v}$ is the coefficient of the $z^{\nu} \Phi_{j}$ term of the expansion of $\Phi_{u}^{\prime}$ in $z$, and the $\left.\Phi_{1}, \ldots, \Phi_{r}.\right)$

Finally, choose $\Phi_{R_{k+1}+1}, \Phi_{R_{k+1}+2}, \cdots, \Phi_{b}$ to be a bas is for $H_{p}^{k+2}$.

Let $H^{\prime}=H_{p}^{k+1}$ and $H^{\prime \prime}$ be the subspace of $H$ spanned by $\Phi_{1}, \ldots, \Phi_{R_{k}}$. Thus $\left.j_{p}^{k}\right|_{H^{\prime \prime}}$ is an isomorphism of $H^{n}$ with $J_{p}^{k}(E)$.

Now, if $p^{\prime}$ is in $G$ we need to know the relationship between $H_{p^{\prime}}^{k+1}$ and $H_{p}^{k+1}$ in order to construct the local coordinates of the $H_{p^{\prime}}^{k+1}$ in the standard chart in $U_{H^{\prime}, H^{\prime \prime}}$. Let $G$ be made small enough so that $H_{p^{\prime}}^{k+1}$ is in $U_{H^{\prime}, H^{n}}$ for all $p^{\prime}$ in $G$. Then, to construct the linear map of $H^{\prime}$ into $H^{\prime \prime}$ corresponding to $H_{p^{\prime}}^{k+1}$ for each $p^{\prime}$ in $G$, we will take a basis of $H_{p^{\prime}}^{k+1}$, split its elements into $H^{\prime}$ and $H^{\prime \prime}$ components, and then map each $H^{\prime}$ component into the corresponding $H^{\prime \prime}$ component. In more detail, for all $v, R_{k} \leq v \leq b$,

$$
\widetilde{\Phi}_{v}=\Phi_{v}+\sum_{u=1}^{R_{k}} Z_{u v}\left(p^{\prime}\right) \Phi_{u}
$$

where the $Z_{u v}\left(p^{\prime}\right)$ are constants chosen so that the $\widetilde{\Phi}_{v}$ lie in $H_{p^{+}}^{k+1}$ and thus will span $H_{p}^{k+1}$. That this is possible is a restatement of the fact that $H_{p^{\prime}}^{k+1}$ and $H$ are complementary direct summands of $H$, that the $\Phi_{v}$ have the $\widetilde{\Phi}_{v}$ as projections in $H_{p^{\prime}}^{k+1}$, and that the $\widetilde{\Phi}_{v}$ span $H_{p^{\prime}}^{k+1}$ since the $\Phi_{u}, 1 \leq u \leq R_{k}$, span $H^{\prime \prime}$. Then the map $H^{\prime} \rightarrow H^{n}$ in $L\left(H^{\prime}, H^{\prime \prime}\right)$ corresponding to $H_{p^{\prime}}^{k+1}$ is defined by linearly extending the map of the basis elements

$$
\Phi_{v} \rightarrow \sum_{u=1}^{R} Z_{u v}\left(p^{\prime}\right) \Phi_{u}, \quad R_{k} \leq v \leq h .
$$

This has the matrix $\left(Z_{u v}\left(p^{\prime}\right)\right)$.

Next, we need to calculate, in the local coordinates $z=\left(z_{1}, z_{2}, \ldots, z_{n}\right)$ centered at $p$, the $n \times R_{k}\left(b-R_{k}\right)$ Jacobian matrix

$$
\left(D^{i} Z_{u v}(p)\right), \quad 1 \leq j \leq n, 1 \leq u \leq R_{k}, R_{k}<v \leq b .
$$

We accomplish this by the technique of implicit differentiation.

First, let

$$
\Phi_{\nu}(q)=\sum_{i=1} \sum_{|\mu| \geq 0} a_{\nu \mu}^{i} z(q)^{\mu} \Phi_{i}(q), \quad 1 \leq v \leq b,
$$

where the convergences are good in (a possibly smaller) $G$. 
Then

$$
\Phi_{\nu}(q)=\sum_{i=1}^{r} \sum_{|\mu| \geq 0} a_{\nu \mu}^{i}(q)^{\mu} \Phi_{i}(q)+\sum_{u=1}^{R_{k}} Z_{u v}(p) \sum_{i=1}^{r} \sum_{|\mu| \geq 0} a_{u \mu} z(q)^{\mu} \Phi_{i}(q)
$$

for $R_{k}<v \leq b$. But $\Phi_{v}(q)$ vanishing of order $k$ at $p^{\prime}$ implies that, for each multi-index $\sigma$, with $|\sigma| \leq k$, each $i$, and each $v, R_{k}<v \leq b$,

$$
\begin{aligned}
0 & =D^{\sigma}\left\{\sum_{|\mu| \geq 0} a_{v \mu}^{i} z(q)^{\mu}+\sum_{u=1} Z_{u v}\left(p^{\prime}\right) \sum_{|\mu| \geq 0} a_{u \mu}^{i} z(q)^{\mu}\right\}\left(p^{\prime}\right) \\
& =\sum_{\mu \geq \sigma} a_{\nu \mu}^{i} C(\mu, \sigma) z\left(p^{\prime}\right)^{\mu-\sigma}+\sum_{u=1} Z_{u \nu}\left(p^{\prime}\right) \sum_{\mu \geq \sigma} a_{u \mu} C(\mu, \sigma) z\left(p^{\prime}\right)^{\mu-\sigma} .
\end{aligned}
$$

Now, differentiate with respect to $z_{j}\left(p^{\prime}\right)$ for each $j, 1 \leq j \leq n$, and evaluate at $p$ to get

$$
\begin{aligned}
0= & \sum_{\mu \geq \sigma} \alpha_{\nu \mu}^{i}\left(\mu_{j}-\sigma_{j}\right) C(\mu, \sigma) z(p)^{\mu-\sigma-\delta}{ }_{j}+\sum_{u=1} D^{j} Z_{u \nu}(p) \sum_{\mu \geq \sigma} a_{u \mu}^{i} C(\mu, \sigma) z(p)^{\mu-\sigma} \\
& +\sum_{u=1}^{R_{k}} Z_{u v}(p) \sum_{\mu \geq \sigma} \alpha_{u \mu}^{i}\left(\mu_{j}-\sigma_{j}\right) C(\mu, \sigma) z(p)^{\mu-\sigma-\delta}{ }_{j} .
\end{aligned}
$$

But, since $Z_{u v}(p)=0$ for all $u, v$ and $z_{j}(p)=0$ for all $j, 1 \leq j \leq n$, we have the equations

$$
0=a_{v \sigma+\delta_{i}}^{i} C\left(\sigma+\delta_{j}, \sigma\right)+\sum_{u=1}^{R_{k}} D^{j} Z_{u v}(p) a_{u \sigma}^{i}
$$

or

$$
0=\left(\sigma_{j}+1\right) a_{\nu \sigma+\delta_{j}}^{i}+\sum_{u=1}^{R_{k}} D^{j} Z_{u \nu}(p) a_{u \sigma}^{i}
$$

satisfied by the components $D^{i} Z_{u v}(p)$ of the Jacobian matrix.

We are interested then in the rank of this Jacobian matrix.

First, by our choice of basis, if $u$ corresponds to the $(n+2)$-tuple $(l,|\lambda|, \lambda)$, then

$$
\alpha_{u \sigma}^{i}= \begin{cases}1 & \text { if } i=l, \lambda=\sigma \\ 0 & \text { otherwise }\end{cases}
$$

so that we get

$$
D^{j} Z_{u \nu}(p)=-\left(a_{j}+1\right) a_{\nu \sigma+\delta_{j}}^{i}
$$

where $u$ corresponds to $(i,|\sigma|, \sigma)$. Also, since $a_{v \sigma+\delta_{j}}^{i}=0$ if $\left|\sigma+\delta_{j}\right|<k+1$, we 
have $D^{j} Z_{u v}(p)=0$ for $u$ corresponding to $(i,|\sigma|, \sigma)$ and any $v$, when $\left|\sigma+\delta_{j}\right|<$ $k+1$, i.e. $|\sigma|<k$.

Finally, when $v>R_{k+1}$ the element $\Phi_{v}$ is in $H_{p}^{k+2}$, and we have $\alpha_{v \sigma_{+} \delta_{j}}^{i}=0$ for $|\sigma| \leq k$.

So the only possible nonzero columns of the Jacobian matrix $\left(D^{j} Z_{u v}(p)\right)$ are those for the pairs $u$ and $v$ with $u$ corresponding to $(i, k, \sigma)$ and $v$ satisfying $R_{k}<v \leq R_{k+1}$. These number

$$
\left(R_{k}-R_{k-1}\right)\left(R_{k+1}-R_{k}\right)=r^{2} \cdot C(n-1+k, n-1) C(n+k, n-1)
$$

in all.

Clearly, if $R_{k+1} \leq b$ and $p$ is not in $W_{k+1}(H)$, there are at least $n$ linearly independent columns, the Jacobian matrix is of rank $n$, and $p$ is not in $S_{k}(H)$. Q.E.D.

From the proof of the theorem we can conclude the following.

Corollary 3.2. If the vector bundle $E$ is in fact a line bundle, and $b \geq R_{1}$, then $S_{0}(H)=W_{1}(H)$.

Corollary 3.3. If the manifold is of dimension $n=1$, and $b \geq R_{k}+1=r k+$ $r+1$, then $S_{k}(H)=W_{k+1}^{u}(H)$ where $u=r k+r+1$. (See $\$ 2$ for the definition of $\left.w_{k+1}^{u}(H).\right)$

Corollary 3.4. If the manifold is of dimension $n=1$, the vector bundle $E$ is is a line bundle, and $b \geq R_{k}+1=k+2$, then $S_{k}(H)=W_{k+1}(H)$.

3.5. Now, if $H$ is equipped with a hermitian metric, $\mathrm{Gr}_{b-R_{k}, R_{k}}(H)$ can be given the Kähler metric $\left(^{*}\right)$, and the pullback $\omega_{k}$ of $\omega$ to $M-W_{k}(H)$ induces on it a Kähler semimetric, positive definite on $\left(M-W_{k}(H)\right)-S_{k}(H)$.

In particular, if $E$ is the canonical line bundle $K$, the vector space $\Gamma(K)$ of its holomorphic sections is equipped with a natural hermitian metric given by

$$
\left\langle\sigma_{1}, \sigma_{2}\right\rangle=\int_{M} \sigma_{1} \wedge \bar{\sigma}_{2}, \quad \sigma_{1}, \sigma_{2} \text { in } \Gamma(k) .
$$

In the case $k=0$, the induced semi-Kähler metric on $M-W_{0}(H)$ is the Kobayashi generalization of the Bergman metric. (See Kobayași $[\mathrm{K}]_{\text {.) }}$

If $M$ is a curve, $E$ is a power of the canonical bundle, and $H$ is a Hilbert space, then the Kähler semimetrics defined by $\omega_{k}$ are those defined by Lewittes. (See [Lw].)

If $M$ is arbitrary and $E$ is of the form $E_{0} \otimes K$ where $E_{0}$ is a holomorphic vector bundle equipped with a hermitian metric, $\Gamma(E)$ can be equipped with a hermitian metric by $\left\langle\sigma_{1}, \sigma_{2}\right\rangle=\int_{M}\left(\sigma_{1}, \sigma_{2}\right), \sigma_{1}, \sigma_{2}$ in $\Gamma(E)$, where $\left(\sigma_{1}, \sigma_{2}\right)$ is the section of $K \otimes \bar{K}$ defined by taking the scalar product in the fibers of $E_{0}$ with values in the fibers of $K \otimes \bar{K}$. 


\section{Examples.}

4.1. Now we examine some examples. The first examples deal with line bundles on Riemann surfaces and we need some preparation.

First, we get a result stronger than those of $\$ 4$, since for Riemann surfaces, the notion of "order of vanishing of a holomorphic function" works well.

So, suppose that $M$ is a compact Riemann surface of genus $g$ and that $E$ is a holomorphic line bundle $L$ on $M$. Then the rank of $J^{k}(L)$ is $R_{k}=1 \cdot C(1+k, 1)$ $=k+1$. Taking the highest case $R_{k}=b$, i.e. $k=b-1$, we have the result that $W_{b-1}(L)$ is the zero set of the section $\wedge^{b} j^{b-1}$ of the line bundle $\left(\Lambda^{b}(M \times H)\right)^{*} \otimes$ $\wedge^{b} J^{b-1}(L)$.

However, in the case of a line bundle, we have the following.

Proposition 4.1 (see [G, p. 103]). If $s$ is a nontrivial bolomorpbic section of the line bundle $L$ over a compact Riemann surface $M$, then for the first Chern number of $L, c_{1}(L)$,

$$
c_{1}(L)=\sum_{p \in M} \nu_{p}(s)
$$

where $\nu_{p}(s)$ is the order of vanishing of $s$ at $p$.

Thus, we have the following:

Proposition 4.2. If $\wedge^{b} j^{b-1}$ is nontrivial then the number of Weierstrass points (including multiplicity) is

$$
\sum_{p \in M} \nu_{p}\left(\bigwedge^{b} j^{b-1}\right)=c_{1}\left(\left(\bigwedge^{b}(M \times H)\right)^{*} \otimes \bigwedge^{b} J^{b-1}(L)\right)=(g-1)(b-1) b+b c_{1}(L) .
$$

Proof. The first equality is Proposition 4.1.

For the second we have, using elementary properties of $c_{1}$, linear algebra, the fact that $c_{1}(M \times H)=0$ since $M \times H$ is trivial, and the exact sequence $(e l)$ from $\$ 1$,

$$
\begin{aligned}
c_{1} & \left(\left(\bigwedge^{b}(M \times H)\right)^{*} \otimes \bigwedge^{b} J^{b-1}(L)\right)=c_{1}\left(\left(\bigwedge^{b}(M \times H)\right)^{*}\right)+c_{1}\left(\bigwedge^{b} J^{b-1}(L)\right)=c_{1}\left(\bigwedge^{b} J^{b-1}(L)\right) \\
& =c_{1}\left(\bigoplus_{i=0}^{b-1}\left(S^{i}(K) \otimes L\right)\right) \quad\left(\text { since } T^{*}-K=\right.\text { canonical line bundle) } \\
& =\sum_{i=0}^{b-1} c_{1}\left(K^{i} \otimes L\right)=(b-1) b c_{1}(K) / 2+b c_{1}(L)=(b-1) b(g-1)+b c_{1}(L) \text { Q.E.D. }
\end{aligned}
$$

Note that this formula depends only on $b$ and not on $H$, except that $\wedge^{b} j^{b-l}$ must be nonzero somewhere.

In the rest of this discussion of examples, we will take $H=\Gamma(E)$, write $\gamma(E)$ for $h$, and write $W_{k}(E)$ for the Weierstrass sets of $M, E$, and $\Gamma(E)$. 
Example 1. $M$ is a compact Riemann surface of genus $g$ and $L=K$ is the canonical bundle of $M$. Here, the definition agrees with the classical definition of Weierstrass points for $k, 0 \leq k \leq g-1$ (see [Lw] and [G]).

The total number of $(g-1)$ th order Weierstrass points for $K$, counting order is, by Proposition 4.2,

$$
(g-1)(g-1) g+2(g-1) g=(g-1) g(g+1),
$$

which agrees with the classical result (see [G, p. 123]).

For future use, we define: An integer $k \geq 1$ is a gap number for a point $\mathrm{p}$ in $M$ if and only if $p$ is not in $W_{k-1}(K)-W_{k-2}(K) \quad\left(W_{-1}(K)=\varnothing\right)$.

There are $g$ gap numbers $\left\{\rho_{i}\right\}, 1 \leq i \leq g$, at a point $p$ which satisfy $1=\rho_{1}$ $<\rho_{2}<\cdots<\rho_{g}<2 g$ (see [G, P. 120]). $p$ is a Weierstrass point if and only if the gap sequence $\left\{\rho_{i}\right\}$ is not $1<2<\cdots<g$. We call the numbers $\sigma_{i}, 1 \leq i \leq g$ which are not gap numbers and which satisfy $1<\sigma_{i} \leq 2 g, \sigma_{i} \leq \sigma_{i+1}$ the nongap numbers of $p$.

4.2. Before dealing with the next examples, which are of arbitrary line bundles, we review some results on line bundles. (We refer mostly to Gunning [G] for proofs. See especially $\$ \$ 7$ and 10.)

Proposition 4.3. Let $L$ be a line bundle on the Riemann surface M. Let $s$ be a nontrivial section of $L$. Then $L$ is isomorphic with the line bundle associated to the divisor of $s$.

Proof. Let $\left\{U_{a}\right\}$ be a covering of $M$ over which $L$ is locally trivialized an $\left\{\phi_{a \beta}\right\}$ be the transition functions for $L$ and $\left\{U_{\alpha}\right\}$. Let $\left\{s_{\alpha}\right\}$ be a local representation for $s$. Thus $s_{\alpha}=\phi_{a \beta} s_{\beta}$ and $\phi_{\alpha \beta}=s_{\alpha} / s_{\beta}$. But $\left\{s_{\alpha} / s_{\beta}\right\}$ is the set of transition functions for the line bundle associated to the divis or of S. Q.E.D.

Using the Riemann-Roch theorem, Gunning has the

Proposition 4.4 [G, pp. 111-112]. (a) If $c_{1}(L)<0$ then $\gamma(L)=0$.

(b) If $c_{1}(L)=0$ and $L=M \times \mathrm{C}$ is the trivial line bundle, then $\gamma(L)=1$.

(c) If $c_{1}(L)=0$ and $L \neq M \times \mathbf{C}$ then $\gamma(L)=0$.

(d) If $0<c_{1}(L)<2 g-2$ then $c_{1}(L)-(g-1) \leq \gamma(L) \leq \min \left(g, c_{1}(L)+1\right)$. of course, bere, $g \neq 0$.

(e) If $c_{1}(L)=2 g-2$ and $L=K$, then $\gamma(L)=g$.

(f) If $c_{1}(L)=2 g-2$ and $L \neq K$, then $\gamma(L)=g-1$. Of course, bere, $g \neq 0$.

(g) If $c_{1}(L)>2 g-2$ then $\gamma(L)=c_{1}(L)-(g-1)$.

Since we are interested only in those $L$ with $\gamma(L) \neq 0$, we rule out (a) and (c). However, in case (a), since $c_{1}(L)=-c_{1}\left(L^{-1}\right)$, we can still study $L$ by studying $L^{-1}$. We remark here that in case (b), we always have $W_{0}(M \times C)=\varnothing$. 
Example 2. If $M$ has genus $g=0$, i.e. $M=\mathbf{P}_{1}$, then all line bundles on $\mathbf{P}_{1}$ are of the form $\zeta^{m}, m \in \mathrm{Z}$, where $\zeta$ is the dual of the tautological line bundle on $P_{1}$, and $c_{1}(\zeta)=1\left[G\right.$, pp. 115-11G]. So $c_{1}\left(\zeta^{m}\right)=m$.

By Proposition 4.2, all $W_{k}\left(\zeta^{m}\right)$ are empty, for $1 \leq k \leq m$.

Example 3. If $g=1$, then for any line bundle $L$, with $c_{1}(L)>0$, case $(g)$ of the proposition gives $\gamma(L)=c_{1}(L)$. Write $d$ for this common positive integer. According to Proposition 2.1, the number of zeros of $\wedge^{d} j^{d-1}$ is $d^{2}$.

If $d=1, L$ must be a line bundle $\zeta_{p}$ associated with a unique (to $L$ ) point divisor $p$ in $M$. Then $W_{0}(L)=\{p\}$, and $W_{k}(L)=\varnothing$ for $k \geq 1$.

In order to discuss the case when $d>1$, we first consider the $m$ th tensor power line bundle $\zeta_{p}^{m}=\left(\zeta_{p}\right)^{m}, m \in \mathbf{Z}, m>1$. Then $c_{1}\left(\zeta_{p}^{m}\right)=m$ and $\gamma\left(\zeta_{p}^{m}\right)=$ $c_{1}\left(\zeta_{p}^{m}\right)=m$, by $(g)$ of Proposition 4.4 .

Proposition 4.5. $p$ is not in $W_{k}\left(\zeta_{p}^{m}\right)$ for all $0 \leq k<m-1$. $p$ is in $W_{m-1}\left(\zeta_{p}^{m}\right)$. $p$ is not in $W_{k}\left(\zeta_{p}^{m}\right)$ for all $k \geq m$.

Proof. $s_{p}^{m}$ is a section in $\Gamma\left(\zeta_{p}^{m}\right)$, which vanishes only at $p$ with order $m$.

Let $k$ be an integer satisfying $k \geq 1$. It is known (see [G, p. 121$]$ ) that there is a meromorphic function $f_{p, k}$ on $M$ whose only singularity is a pole at $p$ of order exactly $k$ if and only if $k$ is a nongap number. On the other hand, since $g=1, M$ does not have any Weierstrass points for the canonical bundle since the total number is $(g+1) g(g-1)$. So the only gap number for any point is 1 . Thus the meromorphic functions $f_{p, k}$ exist for every $p$ and every $k>1$.

So, for $1 \leq k \leq m, f_{p, k} s_{p}$ is a homomorphic section of $\zeta_{p}^{m}$. It vanishes at $p$ of order $m-k$, where $0 \leq m-k<m-1$. Thus $j_{p}^{m-k^{\prime}}: \Gamma\left(\zeta_{p}^{m}\right) \rightarrow J_{p}^{m-k^{\prime}}\left(\zeta_{p}^{m}\right)$ is surjective for all $m-k^{\prime}, 0 \leq m-k^{\prime}<m-1$, and $p$ is not in $W_{m-k^{\prime}}\left(\zeta_{p}^{m}\right)$ for all such $k^{\prime}$.

Since the set of $m$ sections $\left\{s_{p}^{m}, f_{p, 2} s_{p}^{m}, \ldots, f_{p, m} s_{p}^{m}\right\}$ has distinct vanishing order at $p$, they must give a basis for $\Gamma\left(\zeta_{p}^{m}\right)$. Then it is clear that $j^{m-1}$ : $\Gamma\left(\zeta_{p}^{m}\right) \rightarrow J_{p}^{m-1}\left(\zeta_{p}^{m}\right)$ is not surjective and that when $k=m$ and $R_{m}=m+1>$ $\gamma\left(\zeta_{p}^{m}\right)=m$, we have $j_{p}^{m}: \Gamma\left(\zeta_{p}^{m}\right) \rightarrow J_{p}\left(\zeta_{p}^{m}\right)$ is injective so that $p$ is not in $W_{k}\left(\zeta_{p}^{m}\right)$ for $k \geq m$. Q.E.D.

Now, consider an arbitrary line bundle $L$ with $\gamma(L)=c_{1}(L)=d>2$. Let $b$ be any point in $M$.

Consider the line bundle $L \zeta_{b}^{1-d}$, which has $c_{1}\left(L \zeta_{b}^{1-d}\right)=1$. From the case above when $d=1$, we see that there is exactly one $p$ in $M$ such that $L \zeta_{b}^{1-d}=$ $\zeta_{p}$, from which we get $L=\zeta_{p} \zeta_{b}^{d-1}$.

If $p=b$ then $L=\zeta_{b}^{d}$ and from Proposition 4.5,b is not in $W_{k}(L)$ when $k \neq$ $d-1$ and $b$ is in $W_{d-1}(L)$. 
If $p \neq b$ then, letting $s_{p}$ be a nontrivial section of $\zeta_{p}, s_{p}$ does not vanish at $b$ (it vanishes exact by at $p$ ). Thus, considering the basis $s_{b}^{d-1}$, $f_{b, 2} s_{b}^{d-1}, \cdots, f_{b, d-1} s_{b}^{d-1}$, of $\Gamma\left(\zeta_{b}^{d-1}\right)$, we get $d-1$ linearly independent elements $B=\left\{s_{p} s_{b}^{d-1}, s_{p} f_{b, 2} s_{b}^{d-1}, \cdots, s_{p} f_{b, d-1} s^{d-1}\right\}$ of $\Gamma\left(\zeta_{p} \zeta_{b}^{d-1}\right)$. To find a basis for $\Gamma\left(\zeta_{p} \zeta_{b}^{d-1}\right)$ we need to select one more element of $\Gamma\left(\zeta_{p} \zeta_{b}^{d-1}\right)$ which, together with the $d-1$ elements in $B$, forms a linearly independent set. Let $s^{\prime}$ be any section in $\Gamma\left(\zeta_{p} \zeta_{b}^{d-1}\right)$ not lying in the span of $B$ in $\Gamma\left(\zeta_{p} \zeta_{b}^{d-1}\right)$. Then there is an $s$ which is $s^{\prime}$ minus some linear combination of the elements of $B$, which vanishes at $b$ with order $\nu_{b}(s)$ and $\nu_{b}(s)=$ $d-2$ or $\nu_{b}(s)>d-1$. S cannot vanish at $b$ with order larger than $d$ by Proposition 4.1 since then $d=c_{1}(L)=\Sigma \nu_{p}(s) \geq \nu_{b}(s)>d$. So $\nu_{b}(s)=d-2$ or $\nu_{b}(s)=d$. But if $\nu_{b}(s)=d$ then $s$ vanishes only at $b$ and with order $d$, so that by Proposition 4.3, $L=\zeta_{b}^{d}$ which contradicts the assumption $p \neq b$. Thus $\nu_{b}(s)=d-2$.

From knowing $s$ and the set $B$, it is clear that $j_{b}^{k}: \Gamma\left(\zeta_{p} \zeta_{b}^{d-1}\right) \rightarrow$ $J^{k}\left(\zeta_{p} \zeta_{b}^{d-1}\right)$ is surjective for $k \leq d-1$ and injective for $k>d-1$. Thus $b$ is not in any $W_{k}(L)$.

A minor modification of the above yields the same result for $L$ with $d=2$.

Thus we have shown

Proposition 4.6. If $c_{1}(L)=\gamma(L)=d>1$, then for each $b$ in $M$, there is a unique $p$ in $M$ sucb tbat $L=\zeta_{p} \zeta_{b}^{d-1}, b$ is not in $W_{k}(L)$ for $k \neq d-1$, and $b$ is in $w_{d-1}(L)$ if and only if $p=b$.

Example 4. Similar arguments give the following (not as nice) results for line bundles $L$ on Riemann surfaces of genus $g=2$.

If $c_{1}(L)=1$, then $\gamma(L)=0$ or $\gamma(L)=1$ (Proposition 4.2). If $\gamma(L)=1$, then $W_{0}(L)$ consists of one point $p$ and $L=\zeta_{p}$. Also, $W_{k}(L)=\varnothing$ for all $k=0$,

If $c(L)=2$ then either $L$ is or is not the canonical line bundle $K$ of $M$.

If $L=K$, then $\gamma(K)=2$ (Proposition 4.2). From classical results (see [G, p. 229]) we know that $M$ is hyperelliptic, there are six distinct points $w_{i}$, $1 \leq i \leq 6$, in $W_{1}(K)$, and there are no points in $W_{k}(K)$ where $k \neq 1$. We point out also that for each $p$ in $M$, there exists exactly one $q$ in $M$ such that $K=\zeta_{p} \zeta_{q}$, and that $p=q$ if and only if it is in $W_{1}(K)$.

If $c(L)=2$ and $L \neq K$, then $\gamma(L)=1$ (Proposition 4.2) and $L=\zeta_{p} \zeta_{q}$, where $p$ and $q$ are unique. Then for $p \neq q$, we have $W_{0}(L)=\{p, q\}$ and $W_{k}(L)=\varnothing$ for $k \neq 0$. If $p=q$ then $W_{0}(L)=W_{1}(L)=\{p\}$ and $W_{k}(L)=\varnothing$ for $k \neq 0,1$. 
If $c_{1}(L)=d>2$, then $\gamma(L)=d-1$ (Proposition 4.2). Then there are $2(d-1)^{2}$ points in $W_{d-2}(L)$ (counted with multiplicities as zeros of $\wedge^{d-1} j^{d-2}$ ). To find these points, we proceed by noting that, for any point $b$ in $M, L \cdot \zeta_{b}^{2-d}$ has Chern number 2 and $L \cdot \zeta_{b}^{2-d}=\zeta_{p} \zeta_{q}$ where $p$ and $q$ are unique if and only if $\zeta_{p} \zeta_{q}$ is not the canonical bundle. By examining cases, we have the following possibilities.

Either $b$ is not in $W_{d-2}(L)$, or, one of the following occurs:

(1) $L=\zeta_{b}^{d}$ where $b$ is in $W_{1}(K)$. Then $b$ is in $W_{d-3}(L)$ (and hence $\left.W_{d-2}(L)\right)$ and is not in $W_{k}(L), k<d-3$. $b$ is also in $W_{d-1}(L)$ but not in $W_{d}(L)$.

(2) $L=\zeta_{w}^{2} \zeta_{b}^{d-2}$ where $w$ is in $W_{1}(K)$ and $b$ is not in $W_{1}(K)$. Then $b$ is in $W_{d-3}(L)$ (and hence in $\left.W_{d-2}(L)\right)$ but not in $W_{k}(L), k<d-3$. $b$ is not in $W_{d-1}(L)$.

(3) $L=\zeta_{p} \zeta_{b}^{d-1}$ where $p \neq b$ and $\zeta_{p} \zeta_{b} \neq K$. Then $b$ is in $W_{d-2}(L)$ and not in $W_{k}(L), k<d-2$. $b$ is not in $W_{d-1}(L)$.

(4) $L=\zeta_{b}^{d}$ where $b$ is not in $W_{1}(K)$. Then $b$ is in $W_{d-2}(L)$ and not in $W_{k}(L), k<d-2 . \quad b$ is in $W_{d-1}(L)$ and not in $W_{d}(L)$.

If there are $n_{i}$ distinct points $b$ for which $L$ can be represented in case (i) for $1 \leq i \leq 4$, then after examining the vanishing behavior of $\wedge^{d-1} j^{d-2}$ at points of each type, we have

$$
3 n_{1}+2 n_{2}+n_{3}+2 n_{4}=2(d-1)^{2}>0 .
$$

4.4. In the next example, we examine complex analytic manifolds of higher dimension and Weierstrass points for their canonical bundles. The canonical bundle $K$ is but one of a number of bundles whose invariants are important to the manifold in the classification of its complex structure, some others being the tangent bundle $T$, the cotangent bundle $T^{*}$, their products (tensor, symmetric, exterior) and their associated $k$-jet bundles. The Weierstrass point behavior of these bundles ( such as the codimension of the $W_{k}$ ) can help to classify them.

Example 5. We discuss the Weierstrass points of the canonical bundle of a hypersurface in $n+1$ dimensional complex projective space $\mathbf{P}_{n+1}$.

Proposition 4.7. If $V$ is nonsingular (algebraic) irreducible bypersurface of degree $d$ in $\mathbf{P}_{n+1}$, $I$ is the byperplane line bundle on $\mathbf{P}_{n+1}$, and $k_{V}$ is the canonical bundle on $V$, we bave $\Gamma\left(V, k_{V}\right) \simeq \Gamma\left(\mathrm{P}_{n+1}, l^{d-(n+2)}\right)$.

Assume now that $d>n+2$.

The general section of $I^{d-(n+2)}$ is given by a homogeneous polynomial of degree $d-(N+2)$ in the homogeneous coordinates $z_{0}, \cdots, z_{n+1}$ : (using multiindex notation) $s=\Sigma|\mu|=d-(n+2)^{a} z^{\mu}$. To get the representative of $s$ in the local coordinate system on $U^{i}$, the set of points in $\mathbf{P}_{n+1}$ with homogeneous coordinate $Z_{i} \neq 0$, take $s_{i}=s / z_{i}^{d-(n+2)}$. 
The dimension of $\Gamma\left(\mathbf{P}_{n+1}, l^{d-(n+2)}\right)$ is thus $\gamma\left(k_{V}\right)=C(n+m+1, n+1)$ $=C(d-1, n+1)$, where $m=d-(n+2)$.

Then, we have the

Proposition 4.8. For $k \leq m, R_{k}=C(n+k, n)<\gamma\left(k_{V}\right)=C(n+m+1, n+1)$ and $W_{k}(V)=\varnothing$.

The next step then is to consider whether a $p$ in $M$ is in $W_{m+1}\left(k_{V}\right)$.

To examine this further, consider the homogeneous polynomial degree $d$, $P(z)=\sum_{i=0}^{n+1} z_{i}^{d}$, where $d>n+2$. After some work, we have the following results:

If $n=1$, and $d=4$, then $m=1$ and we have $W_{0}\left(k_{V}\right)=W_{1}\left(k_{V}\right)=\varnothing, W_{2}\left(k_{V}\right)$ $=\{\langle 1,0, \alpha\rangle,\langle 0,1, \alpha\rangle,\langle 1, \alpha, 0\rangle$ : where $d$ is a fourth root of -1$\} . R_{2}=3=\gamma\left(k_{v}\right)$.

If $n=2$, and $d=5$, then $m=1$ and we have $w_{0}\left(k_{V}\right)=w_{1}\left(k_{V}\right)=\varnothing, w_{2}\left(k_{V}\right)=$ $\{\langle 1,0,0, a\rangle,\langle 1,0, a, 0\rangle,\langle 1, a, 0,0\rangle,\langle 0,1,0, a\rangle,\langle 0,1, a, 0\rangle,\langle 0,0,1, a\rangle$ : where $\alpha$ is a fifth root of -1$\}$. Here, $R_{2}=6>\gamma\left(k_{V}\right)=4>R_{1}=3$.

If $n=2$, and $d=6$, then $m=2$ and we have $W_{0}\left(k_{V}\right)=W_{1}\left(k_{V}\right)=W_{2}\left(k_{V}\right)=\varnothing$, and $W_{3}\left(k_{V}\right)$ is a reducible divisor. Here $R_{3}=10=\gamma\left(k_{V}\right)$.

4.5. Example 6. In this example, we note that

Proposition 4.9. The tangent bundle of projective space of $n$ dimension bas $W_{k}\left(T \mathbf{P}_{n}\right)=\varnothing$ for all $k$.

Furthermore, we remark that if $M$ is a homogeneous space and $E$ is a homogeneous vector bundle over $M$, then for any invariant subspace $H$ of $\Gamma(E)$, for example $\Gamma(E)$, we have $W_{k}(H)$ is equal to either all of $M$ or the empty set.

\section{BIBLIOGRA PHY}

[G] R. C. Gunning, Lectures on Riemann surfaces, Princeton Math. Notes, Princeton Univ. Press, Princeton, N. J., 1966. MR 34 \#7789.

[K] S. Kobayashi, Geometry of bounded domains, Trans. Amer. Math. Soc. 92 (1959), 267-290. MR $22 \# 3017$.

[Lw] J. Lewittes, Differentials and metrics on Riemann surfaces, Trans. Amer. Math. Soc. 139 (1969), 311-318. MR 38 \#6050.

[Pa] R. Pala is, Seminar on the Atiyah-Singer index theorem, Ann. of Math. Studies, no. 57, Princeton Univ. Press, Princeton, N. J., 1965. MR 33 \#6649.

DEPARTMENT OF MATHEMATICS, UNIVERSITY OF CALIFORNIA, SAN DIEGO, LA JOLLA, CALIFORNIA 92307 